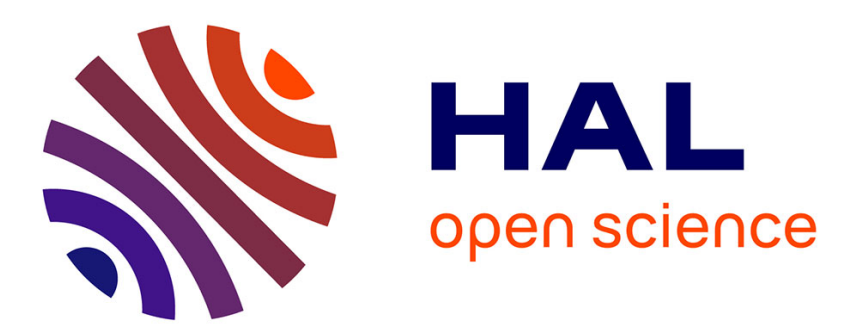

\title{
Leader-Follower Formation Saturated Control for Multiple Quadrotors with Switching Topology
}

\author{
Zhicheng Hou, Isabelle Fantoni
}

\section{To cite this version:}

Zhicheng Hou, Isabelle Fantoni. Leader-Follower Formation Saturated Control for Multiple Quadrotors with Switching Topology. IEEE Workshop on Research, Education and Development of Unmanned Aerial Systems (RED UAS 2015), Nov 2015, Cancún, Mexico. pp.8-14. hal-01264292

\section{HAL Id: hal-01264292 \\ https://hal.science/hal-01264292}

Submitted on 29 Jan 2016

HAL is a multi-disciplinary open access archive for the deposit and dissemination of scientific research documents, whether they are published or not. The documents may come from teaching and research institutions in France or abroad, or from public or private research centers.
L'archive ouverte pluridisciplinaire HAL, est destinée au dépôt et à la diffusion de documents scientifiques de niveau recherche, publiés ou non, émanant des établissements d'enseignement et de recherche français ou étrangers, des laboratoires publics ou privés. 


\title{
Leader-Follower Formation Saturated Control for Multiple Quadrotors with Switching Topology
}

\author{
Zhicheng HOU and Isabelle FANTONI
}

\begin{abstract}
This paper presents a distributed saturated formation control for a multiple quadrotors (UAVs) system with leader-follower structure. Each UAV has local and limited neighbors. Stability analysis for the multi-UAV system with fixed and switching topology is given, using the theories of convex hull and perturbed matrix. Simulation results show that the quadrotors with the proposed strategy can achieve consensus in presence of changeable leaders with switching topology. The real-time experiment shows that the quadrotors can track the desired formation trajectory by using the proposed formation strategy.
\end{abstract}

\section{INTRODUCTION}

The cooperative control of the leader-follower (L-F) multiagent systems has recently attracted the attention. Within the field of mobile robotics, L-F formations arise in applications ranging from searching, surveillance, inspection, and exploration [1]. The formation of multiple UAVs (quadrotors for example) is considered by several papers. In [2], the generation of the formation trajectory is developed. In [3], each quadrotor can obtain the error of position of the group from the prescribed trajectory. In our work, a multiple UAVs (quadrotors) system is considered. The main objective is to design a distributed control law for each UAV, using local sensing to its neighbors, such that all the UAVs can achieve L-F consensus and maintain some formation shapes. A UAV, although the leader, senses its neighbors instead of all the UAVs in the formation. The L-F formation problem is treated in large amount of papers, but in most of them, for example papers [4][5], the leader is treated as a special individual whose motion is independent of other agents. In our work, each leader has interactions with some other UAVs (leaders or followers) which appear in its neighborhood.

The dynamics of a quadrotor has a double-loop property, i.e. the translational (outer loop) and rotational (inner loop) dynamics. The outputs of the translational controller are the inputs of the attitude angles. There are numerous papers or reports, which focus on attitude stabilization of a quadrotor, such as [10][11]. Therefore, in order to focus on our subject, we assume that in this paper, the attitude angles (innerloop states) can track some bounded input, with using some

\footnotetext{
Zhicheng HOU and Isabelle FANTONI are with Sorbonne universités, Université de technologie de Compiègne, CNRS, UMR 7253 Heudiasyc, 60200 Compiègne, France. zhicheng.hou/isabelle.fantoniehds.utc.fr

This work was carried out in the framework of the Labex MS2T, which was funded by the French Government, through the program "Investments for the future" managed by the National Agency for Research (Reference ANR-11-IDEX-0004-02) and the French project SEARCH funded by the Picardie Region Council.
}

attitude controllers. Then, we propose a distributed formation control with saturations.

The switches of the topology of multi-robot systems occur in some cases [6][7]. In this paper, we are concerned by the switch topology caused by the change of leaders in a formation of quadrotors. Researchers, such as in [4][5][8], have proven that the consensus of agents can be achieved with jointly connected graphs. Different from the foregoing mentioned works, we consider a formation with arbitrary switching topology. It has been proved that the switched system with an arbitrary switching law is stable if and only if a common Lyapunov function exists [14]. Therefore, we need that each subgraph is connected instead of jointly connected.

The contributions of this paper can be summarized as follows: the theories of convex hull [12] and perturbed matrix [13] are used to prove the stability of the multi-UAV system, which transforms the stability problem of a nonlinear system (caused by the saturated controller) into the stability problem of a linear time-varying system. Additionally, the common Lyapunov function approach is used to prove the stability of the multi-quadrotor system with arbitrary switching topology.

The paper is organized as follows. Basic concepts in graph theory are presented in section II. The mathematical model of the L-F formation of quadrotors is given in section III. The distributed saturation control is proposed in section IV. The stability analysis is given in section V. Some results of simulation and a real-time experiment are shown in section VI. Finally, some conclusions are stated in section VII.

\section{PRELIMINARIES}

\section{A. Graph theory and notations}

The presentation here is based on material in [5] and [9]. A graph $\mathcal{G}=(\mathcal{V}, \mathcal{E})$ is composed of vertex and edge sets. The vertex set $\mathcal{V}=\{1,2, \ldots, n\}$ represents the indices of UAV $1,2, \ldots, n$. Then, we have $|\mathcal{V}|=n$, where $|\cdot|$ represents the cardinality of a set. The edge set satisfies $\mathcal{E}=\{(i, j): i, j \in$ $\mathcal{V}\}$. A graph is simple if it has no self-loops or repeated edges. In this paper, we have simple graphs. A graph $\mathcal{G}$ is connected, if there is a path between any two vertices, otherwise it is disconnected. We define a set $\mathcal{N}_{i}=\{j \in$ $\mathcal{V}:(i, j) \in \mathcal{E}\}$ that represents the set of labels of UAVs which are neighbors of UAV $i(i \in \mathcal{V})$. We also call $\mathcal{N}_{i}$ the neighbors set of $i$. The adjacency matrix of $\mathcal{G}$ is denoted by $G^{A}=\left[\omega_{i j}^{a}\right] \in R^{n \times n}$, where $\omega_{i i}^{a}=0$, and $\omega_{i j}^{a} \geq 0\left(\omega_{i j}^{a}>0\right.$ if $j \in \mathcal{N}_{i}$ ). Its degree matrix $\mathcal{I}_{n}=\operatorname{diag}\left\{\sum_{j=1}^{n} \omega_{i j}^{a}, i \in \mathcal{V}\right\}$. Then, 
the Laplacian of the graph is defined as

$$
L=\mathcal{I}_{n}-G^{A}
$$

In this paper, we are concerned by the UAVs formation with L-F structure, where we use $\tilde{\mathcal{G}}$ to represent such a LF formation. The graph $\tilde{\mathcal{G}}$ inherits all the definitions of $\mathcal{G}$ as stated before. We define an "interaction matrix", which is based on the Laplacian matrix, to represent the interaction of the UAVs. Firstly, we introduce a diagonal "leader matrix" $G^{l}=\operatorname{diag}\left\{\ldots, \omega_{i i}^{l}, \ldots\right\} \in R^{n \times n}$, where $\omega_{i i}^{l}>0$ if $i$ is a leader and $\omega_{i i}^{l}=0$ if $i$ is a follower. Now we are ready to give the expression of the interaction matrix $G$ as follows

$$
G=L+G^{l}
$$

The weights " $\omega_{i j}^{a}$ " in the adjacent matrix $G^{A}$ are given as follows.

$$
\omega_{i j}^{a}= \begin{cases}1 & \text { if } j \in \mathcal{N}_{i} \\ 0 & \text { otherwise }\end{cases}
$$

The weights " $\omega_{i i}^{l}$ " in the leader matrix $G^{l}$ are given as follows

$$
\omega_{i i}^{l}=\left\{\begin{array}{cc}
1 & \text { if UAV } \mathrm{i} \text { is a leader } \\
0 & \text { otherwise }
\end{array}\right.
$$

Since undirected graphs are considered, then, $G$ is symmetric.

In this paper, we are concerned by a cooperation with switching interconnection topologies, we suppose that there is an infinite sequence of non-overlapping, continuous timeintervals $T_{\gamma}=\left[t_{\gamma}, t_{\gamma+1}\right), \gamma=0,1, \cdots$. We define a finite set $\Gamma=\left\{\tilde{\mathcal{G}}_{\gamma}: \gamma=0,1 \cdots\right\}$, which is the sequence of subgraphs during a formation.

We define a switching signal $\mathcal{S}:\left\{T_{0}, T_{1}, \cdots\right\} \rightarrow \Gamma$ to describe the evolution of the topologies, which is piecewiseconstant (shown in Fig.1 for example).

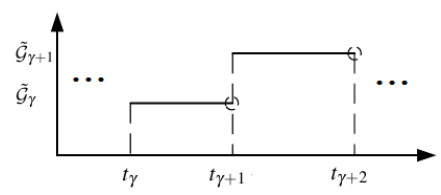

Fig. 1. Switching signal $\mathcal{S}$, on the vertical axis, $\tilde{\mathcal{G}}_{\gamma}$ and $\tilde{\mathcal{G}}_{\gamma+1}$ are two subgraphs. The horizontal axis represents the time $t$. $t_{\gamma}, t_{\gamma+1}$, and $t_{\gamma+2}$ represent some switching time instances.

We denote by $G_{\gamma}$ the interaction matrix of subgraph $\tilde{\mathcal{G}}$. Apparently, the interaction matrix $G_{\gamma}$ changes also with respect to " $\gamma$ ".

We denote by $\sigma_{b}: R \times R^{m} \rightarrow R^{m}$ the saturation function with

$$
\sigma_{b}(u)=\left[\sigma_{b}\left(u_{1}\right), \sigma_{b}\left(u_{2}\right), \cdots, \sigma_{b}\left(u_{m}\right)\right]^{T}
$$

where $\sigma_{b}\left(u_{i}\right)=\operatorname{sgn}\left(u_{i}\right) \min \left\{b,\left|u_{i}\right|\right\}$. The notation "sgn(.)" represents the sign function.

\section{MATHEMATICAL MODELING}

\section{A. System structure}

A multi-UAV system is composed of $n$ autonomous UAVs. Each UAV has a distributed formation controller.

The dynamics of a quadrotor can be considered as a "singular perturbation system"[10]. Thus, it can be divided into rotational (fast) and translational (slow) dynamics. To simplify the model of a quadrotor and focus on our concerns, we assume that the rotational dynamics are fast stabilized, and the attitude angles $\left(\phi_{i}, \theta_{i}\right.$, and $\left.\psi_{i}\right)$ are able to track some bounded references with negligible tracking errors. Thus, we have

$$
\theta_{i} \approx \theta_{i}^{r}, \quad \phi_{i} \approx \phi_{i}^{r}, \quad \text { and } \psi_{i} \approx \psi_{i}^{r}
$$

where $\phi_{i}^{r}$ and $\theta_{i}^{r}$ represent the reference roll and pitch angles for UAV $i$.

To realize the formation control of multiple UAVs, the translational dynamics, which are shown in equations (2) (see [11] and [15] for details), are considered.

$$
\begin{aligned}
& \ddot{X}_{i}=\left(\sin \psi_{i} \sin \phi_{i}+\cos \psi_{i} \sin \theta_{i} \cos \phi_{i}\right) \frac{F_{T}}{m} \\
& \ddot{Y}_{i}=\left(-\cos \psi_{i} \sin \phi_{i}+\sin \psi_{i} \sin \theta_{i} \cos \phi_{i}\right) \frac{F_{T}}{m} \\
& \ddot{Z}_{i}=-g+\left(\cos \theta_{i} \cos \phi_{i}\right) \frac{F_{T}}{m}
\end{aligned}
$$

where $F_{T}$ represents the thrust and $m$ represents the mass of the UAV. We refer to $X_{i}, Y_{i}$ as the position states in the global frame, $\dot{X}_{i}, \dot{Y}_{i}$ as the velocity states.

Since the attitude angles are kept around zero such that $\cos \theta_{i} \cos \phi_{i} \approx 1$, the controller design for $Z$ is trivial. In our work, the formation begins after the altitude is stabilized. Therefore, during the formation, it is feasible to assume that $F_{T} \approx m g /\left(\cos \theta_{i} \cos \phi_{i}\right)$. According to (1), the simplified planar translational dynamics of UAV $i, i \in \mathcal{V}$ w.r.t a global frame can be rewritten as follows

$$
\begin{aligned}
& \ddot{X}_{i}=\left(\sin \psi_{i}^{r} \frac{\tan \phi_{i}^{r}}{\cos \theta_{i}^{r}}+\cos \psi_{i}^{r} \tan \theta_{i}^{r}\right) g \\
& \ddot{Y}_{i}=\left(-\cos \psi_{i}^{r} \frac{\tan \phi_{i}^{r}}{\cos \theta_{i}^{r}}+\sin \psi_{i}^{r} \tan \theta_{i}^{r}\right) g
\end{aligned}
$$

For a quadrotor with fixed yaw angle $\left(\psi_{i}=0\right)$, the translational movement is produced by using pitch and roll angles. Let us define

$$
\begin{aligned}
& \theta_{i}^{r}=\arctan \left(\frac{u_{i}^{X}}{g}\right) \\
& \phi_{i}^{r}=\arctan \left(\frac{-u_{i}^{Y} \cos \left(\arctan \left(u_{i}^{X} / g\right)\right)}{g}\right) \\
& \boldsymbol{\psi}_{i}^{r}=0
\end{aligned}
$$

If we replace $\theta_{i}^{r}$ and $\phi_{i}^{r}$ in (3) by equation (4) and we obtain the planar dynamics in the following decoupled form:

$$
\ddot{X}_{i}=u_{i}^{X}, \quad \ddot{Y}_{i}=u_{i}^{Y}
$$

We just consider the dynamics along one axis ( $X$ for example). The result on the other axis can be obtained with the same analysis. 


\section{B. Overall model for $U A V s$}

In the following part, we will present the model of cooperation of multiple UAVs. First, we define a full states vector as $\mathrm{x}=\left[X_{1}, \ldots, X_{n}, \dot{X}_{1}, \ldots, \dot{X}_{n}\right]^{T}$, where $X_{i}$ represents the position state, while $\dot{X}_{i}$ represents the velocity state. We denote by $\mathrm{u}=\left[u_{1}, \ldots, u_{n}\right]^{T}$ the full input vector, where $n$ is the number of UAVs. It is clear that $\mathrm{x} \in \mathbb{R}^{2 n}$ while $\mathrm{u} \in \mathbb{R}^{n}$.

We denote a constant matrix $I_{N}$ as an identity matrix of size $N \times N$. We also denote a vector $\mathbf{1}_{M} \in \mathbb{R}^{M}$ of size $M$ with all the entries equivalent to 1 . Each UAV makes the measurements of relative positions and velocities with respect to its neighbors, which can be represented by vectors $\left(\begin{array}{c}X_{i}-X_{j} \\ \dot{X}_{i}-\dot{X}_{j}\end{array}\right), j \in \mathcal{N}_{i}$. If the UAV is a leader, besides the foregoing measurements, it can also obtain the relative position and velocity with respect to the reference trajectory $\left(\begin{array}{c}X_{i}-r_{X}(t) \\ \dot{X}_{i}-\dot{r}_{X}(t)\end{array}\right)$, where we denote by $r(t)=\left[r_{X}(t), \dot{r}_{X}(t)\right]^{T}$ to represent the reference trajectory.

We use these measurements to form an "error measurement" (we follow the same notion in [9]), shown by equation (5), the selection of the weights is detailed in section II.

$$
y_{i}=\sum_{j=1}^{n} \omega_{i j}^{a}\left(\begin{array}{c}
X_{i}-X_{j} \\
\dot{X}_{i}-\dot{X}_{j}
\end{array}\right)+\omega_{i i}^{l}\left(\begin{array}{c}
X_{i}-r_{X}(t) \\
\dot{X}_{i}-\dot{r}_{X}(t)
\end{array}\right)
$$

We assume that the reference signal given to the leader (or leaders) is slowly changing such that we have $\ddot{r}_{X} \approx 0$ and $\ddot{r}_{Y} \approx 0$.

Equation (5) can be represented by the following general form

$$
\begin{aligned}
y_{i} & =\left(\sum_{j=1}^{n} \omega_{i j}^{a}+\omega_{i i}^{l}\right)\left(\begin{array}{c}
X_{i} \\
\dot{X}_{i}
\end{array}\right)-\sum_{j=1}^{n} \omega_{i j}^{a}\left(\begin{array}{c}
X_{j} \\
\dot{X}_{j}
\end{array}\right)-\omega_{i i}^{l}\left(\begin{array}{c}
r_{X}(t) \\
\dot{r}_{X}(t)
\end{array}\right) \\
& =\left(I_{2} \otimes\left(\mathrm{g}_{i}^{a}+\mathrm{g}_{i}^{l}\right)\right)^{T} \mathrm{X}-\left(I_{2} \otimes \mathrm{g}_{i}^{l}\right)^{T} \cdot\left(r(t) \otimes \mathbf{1}_{n}\right)
\end{aligned}
$$

where " $\otimes$ " represents the Kronecker product. In the right side of $y_{i}$, the first weights vector satisfies $\mathrm{g}_{i}^{a}=$ $\left[-\omega_{i 1}^{a}, \ldots,-\omega_{i(i-1)}^{a}, \sum_{j=1}^{n} \omega_{i j}^{a},-\omega_{i(i+1)}^{a} \cdots-\omega_{i n}^{a}\right]^{T}$. The second weights vector satisfies $\mathrm{g}_{i}^{l}=\left[0, \ldots, 0, \omega_{i i}^{l}, 0, \ldots, 0\right]^{T}$.

We can observe that $\left[\mathrm{g}_{1}^{a}, \ldots, \mathrm{g}_{n}^{a}\right]^{T}=L$ and $\left[\mathrm{g}_{1}^{l}, \ldots, \mathrm{g}_{n}^{l}\right]^{T}=$ $G^{l}$. Then, if we denote $\mathrm{y}=\left[y_{1}, \ldots, y_{n}\right]^{T}$, we will have the dynamics of the multi-UAV system as follows

$$
\left\{\begin{array}{l}
\dot{\mathrm{x}}=\mathcal{A} \mathrm{x}+\mathcal{B} \mathrm{u} \\
\mathrm{y}=\left(I_{2} \otimes G\right) \mathrm{x}-\left(I_{2} \otimes G^{l}\right)\left(r(t) \otimes \mathbf{1}_{n}\right)
\end{array}\right.
$$

where $\mathrm{y} \in \mathrm{R}^{2 n}$ represents the error measurements of all the UAVs. Matrices $\mathcal{A}, \mathcal{B} \in \mathrm{R}^{2 n \times 2 n}$ have the following expressions

$$
\mathcal{A}=\left(\begin{array}{cc}
\mathbf{0}_{n \times n} & I_{n} \\
\mathbf{0}_{n \times n} & \mathbf{0}_{n \times n}
\end{array}\right) \text { and } \mathcal{B}=\left(\begin{array}{c}
\mathbf{0}_{n \times n} \\
I_{n}
\end{array}\right)
$$

where $\mathbf{0}_{n \times n}$ represents the zero matrix of size $n \times n$.

In the formation with switching topology, the Laplacian matrix $L$ and the leader matrix $G^{l}$ are variable.

\section{DISTRIBUTED SATURATED FORMATION CONTROL}

In this section, we will investigate the formation controller design of the multi-UAV system.

Definition 1: The L-F consensus of system (7), is said to be achieved if, for each UAV $i, i \in \mathcal{V}$, there is a distributed control law $u_{i}$, such that the closed-loop system satisfies

$$
\lim _{t \rightarrow \infty}\left|X_{i}-r_{X}-d_{i 0}\right|=0, \quad i=1, \ldots, n
$$

for some initial condition $x_{i}(0), i=1, \ldots, n$. We denote by $\tilde{X}_{i}$ the desired position of UAV $i$, which satisfies $\tilde{X}_{i}=r_{X}+d_{i 0}$.

The constant scalar $d_{i 0}$ represents the desired inter-distance of UAV $i$ and the reference signal with respect to $X$-axis. The terms $d_{i 0}$ is designed to form some desired formation patterns. It is important to note that $d_{i 0}$ is not available for the followers, who have no knowledge of the reference trajectory.

After the L-F consensus have achieved, it satisfies $d_{i 0}=$ $\tilde{X}_{i}-r_{X}$. Similarly, for $j \in \mathcal{N}_{i}$, we also have $d_{j 0}=\tilde{X}_{j}-r_{X}$ after L-F consensus have been achieved. If we define $d_{0 i}=r_{X}-\tilde{X}_{i}$ and $d_{i j}=\tilde{X}_{i}-\tilde{X}_{j}$. Then, we obtain

$$
d_{i j}=d_{i 0}+d_{0 j}=d_{i 0}-d_{j 0}
$$

where $d_{i j}$ is the constant desired inter-distance of UAV $i$ and its neighboring UAV $j$ (along $X$-axis).

If we define error $e_{i}=X_{i}-r_{X}-d_{i 0}$ and $\dot{e}_{i}=\dot{X}_{i}-\dot{r}_{X}$, the full error vector is given by $\mathrm{e}=\left[e_{1}, \cdots, e_{n}, \dot{e}_{1}, \cdots, \dot{e}_{n}\right]^{T}$. We can write the error dynamics for the overall system as follows

$$
\dot{\mathrm{e}}=\mathcal{A} \mathrm{e}+\mathcal{B} \mathrm{u}
$$

Note that $\ddot{r}_{X} \approx 0$ and $\ddot{r}_{Y} \approx 0$. Notation $\mathrm{u}=\left[u_{1}, \ldots, u_{n}\right]^{T}$ represents the overall control inputs of UAVs. According to definition 1, the consensus of the L-F formation corresponds to the convergence analysis of system (10) origin.

We propose a switching distributed saturated control law $u_{i}$ for UAV $i$ (either a leader or a follower) as follows

$$
u_{i}=-\sigma_{b}\left(K\left(y_{i}-\left(\begin{array}{c}
\sum_{j=1}^{n} \omega_{i j}^{a} d_{i j}+\omega_{i i}^{l} d_{i 0} \\
0
\end{array}\right)\right)\right)
$$

where $K=\left[k_{1}, k_{2}\right]$ is the gain vector. The weights $\omega_{i i}^{l}$ are used to assign a UAV as a leader or as a follower. For instance, if we need UAV $i$ to take the role of leader, we set $\omega_{i i}^{l}=1$, otherwise, we set $\omega_{i i}^{l}=0$.

Remark 1: We note that the switch in the controller $u_{i}$ contains two cases, i) the switch of $\omega_{i j}^{a}$, which can be caused by the sensing/detecting failure; ii) the switch of $\omega_{i i}^{l}$, which is caused by the reassignment of the leaders. The former case is passive, such that we cannot control. The latter case is active, which we are able to control.

\section{Stability ANALYSis}

To analyze the stability of the system (10) by using our proposed formation controller, we use the convex hull and perturbed matrix theories. The formation with fixed and switching topology is considered. The common Lyapunov function method is used for the formation with arbitrary switching. 


\section{A. Fixed topology}

We replace $y_{i}$ by equation (5), the formation controller (11) is rewritten as follows

$$
u_{i}=-\sigma_{b}\left(K\left(\sum_{j=1}^{n} \omega_{i j}^{a}\left(\begin{array}{c}
X_{i}-X_{j}-d_{i j} \\
\dot{X}_{i}-\dot{X}_{j}
\end{array}\right)+\omega_{i i}^{l}\left(\begin{array}{c}
X_{i}-r_{X}-d_{i 0} \\
\dot{X}_{i}-\dot{r}_{X}
\end{array}\right)\right)\right)
$$

Using (9), we obtain

$$
u_{i}=-\sigma_{b}\left(K\left(\left(1+\omega_{i i}^{l}\right)\left(\begin{array}{c}
e_{i} \\
\dot{e}_{i}
\end{array}\right)-\sum_{j=1}^{n} \omega_{i j}^{a}\left(\begin{array}{c}
e_{j} \\
\dot{e}_{j}
\end{array}\right)\right)\right)
$$

Then, according to the definition of the saturation function in section II, we can rewrite the overall control input $u$ as

$$
\mathrm{u}=-\sigma_{b}\left(\left(K \otimes I_{n}\right) \cdot\left(I_{2} \otimes G\right) \cdot \mathrm{e}\right)
$$

Note that $\mathrm{u} \in R^{n}$.

We replace $u$ in (10) by (13), then, we obtain

$$
\dot{\mathrm{e}}=\left(\begin{array}{cc}
\mathbf{0} & I_{n} \\
\mathbf{0} & \mathbf{0}
\end{array}\right) \mathrm{e}-\left(\begin{array}{c}
\mathbf{0} \\
I_{n}
\end{array}\right) \cdot \sigma_{b}\left(\left[\begin{array}{ll}
k_{1} G & k_{2} G
\end{array}\right] \cdot \mathrm{e}\right)
$$

We observe that when the control inputs $u_{1}, \ldots, u_{n}$ are all unsaturated, the foregoing system will become an ordinary linear system as $\dot{\mathrm{e}}=\mathcal{A}_{c} \mathrm{e}$, where $\mathcal{A}_{c}$ is represented as follows

$$
\mathcal{A}_{c}=\left(\begin{array}{cc}
\mathbf{0} & I_{n} \\
-k_{1} G & -k_{2} G
\end{array}\right)
$$

Recall that $G$ is symmetric, we should firstly make sure that the interaction matrix $G$ is positive definite. We have proved that if the graph of the formation of UAVs is connected, the eigenvalues of the interaction matrix always have positive real part [16]. Then, $G$ is positive definite. We can select proper gains $k_{1}$ and $k_{2}$ to assign the poles such that the origin of system (10) is asymptotically stable. It is not difficult to verify that there exists a matrix $\mathcal{P} \succ 0$, which renders $\mathcal{A}_{c}^{T} \mathcal{P}+\mathcal{P} \mathcal{A}_{c} \prec 0$, where notations " $\succ$ " and " $\prec$ " represent that a matrix is "positive-definite" and "negative-definite". If we select a Lyapunov function $V=\mathrm{e}^{T} \mathcal{P} \mathrm{e}$, then, we have $\dot{V}<0$, when e $\neq 0$.

We define a set $\Omega(\mathcal{P}, \rho)=\left\{\mathrm{e} \in R^{2 n}: \mathrm{e}^{T} \mathcal{P} \mathrm{e} \leq \rho\right\}$. Then, $\Omega(\mathcal{P}, \rho)$ is said to be invariant if $\dot{V}<0$ for all e $\in \Omega(\mathcal{P}, \rho)-$ $\{0\}$.

For some selected gains $k_{1}, k_{2}$, the domain of attraction of the equilibrium point (origin) of system (14) is estimated in the sequel. We employ the definition of a diagonal matrix $\Phi_{s}$ in [12]. We define the set $\ell=\left\{l \in R^{n}: l_{i} \in\{1,2\}\right\}$, which contains $2^{n}$ vectors and use an $l \in \ell$ to define a diagonal matrix $\Phi_{s}$ such that

$$
\Phi_{s}(l)=\operatorname{diag}\left\{\delta\left(l_{1}-s\right), \delta\left(l_{2}-s\right), \ldots, \delta\left(l_{n}-s\right)\right\}, s \in\{1,2\}
$$

where $\delta\left(l_{i}-s\right)=1$, if $l_{i}=s$, otherwise, $\delta\left(l_{i}-s\right)=0$. We note that the notation $l_{i}$, which is a scalar, represents the $i$-th entry of vector $l$. In the following part, we use the notation $l_{(\kappa)}$, which is a vector, to represent the $\kappa$-th member of the set $\ell$, thus, $l_{(\kappa)} \in \ell$.

We define a matrix $\mathcal{H} \in R^{n \times 2 n}$, which satisfies $\mathcal{H}^{T} \mathcal{H} \succ 0$, let $H_{i}$ represent the $i$-th row of $\mathcal{H}$. We assume that $\left|H_{i} \mathrm{e}\right| \leq b$, $i \in \mathcal{V}$ for all e $\in \Omega(\rho)$. Then, using lemma 2.3 of [12], we obtain that the control inputs "u" is in the convex hull as follows

$$
\begin{aligned}
& \mathrm{u}=-\sigma_{b}\left(\left[\begin{array}{ll}
k_{1} G & k_{2} G
\end{array}\right] \cdot \mathrm{e}\right) \\
& \in \operatorname{co}\left\{-\Phi_{1}(l) \mathcal{H} \mathrm{e}-\Phi_{2}(l)\left(\left[\begin{array}{ll}
k_{1} G & k_{2} G
\end{array}\right] \cdot \mathrm{e}\right): l \in \ell\right\}, \forall \mathrm{e} \in \Omega(\rho)
\end{aligned}
$$

Then, according to the definition of convex hull, we conclude that for $l_{(\kappa)} \in \ell, u=-\sum_{\kappa=1}^{2^{n}} \alpha_{\kappa}\left(\Phi_{1}\left(l_{(\kappa)}\right) \mathcal{H} \mathrm{e}+\right.$ $\left.\Phi_{2}\left(l_{(\kappa)}\right)\left(\left[\begin{array}{ll}k_{1} G & k_{2} G\end{array}\right] \cdot \mathrm{e}\right)\right)$, where $\alpha_{\kappa}, \kappa \in\{1, \ldots, 2 n\}$ are some nonnegative scalars. They satisfy $\sum_{\kappa=1}^{2^{n}} \alpha_{\kappa}=1$. Then, the error dynamics (10) in closed-loop form can be rewritten as $\dot{\mathrm{e}}=\tilde{\mathcal{A}}_{c} \mathrm{e}$, where $\tilde{\mathcal{A}}_{c}$ is represented as follows

$$
\tilde{\mathcal{A}}_{c}=\left(\mathcal{A}-\mathcal{B} \sum_{\kappa=1}^{2^{n}} \alpha_{\kappa}\left(\Phi_{1}\left(l_{(\kappa)}\right) \mathcal{H}+\Phi_{2}\left(l_{(\kappa)}\right)\left(\left[\begin{array}{ll}
k_{1} G & k_{2} G
\end{array}\right]\right)\right)\right)
$$

If we represent the matrix $\mathcal{H}=\left[\begin{array}{ll}\mathcal{H}_{1} & \mathcal{H}_{2}\end{array}\right]$ in a partitioned form, where $\mathcal{H}_{1}, \mathcal{H}_{2} \in R^{n \times n}$, we can rewrite the foregoing matrix as follows

$$
\tilde{\mathcal{A}}_{c}=\left(\begin{array}{cc}
\mathbf{0} & I_{n} \\
-\tilde{\Phi}_{1} \mathcal{H}_{1}-\tilde{\Phi}_{2} k_{1} G & -\tilde{\Phi}_{1} \mathcal{H}_{2}-\tilde{\Phi}_{2} k_{2} G
\end{array}\right)
$$

where we abbreviate $\sum_{\kappa=1}^{2^{n}} \alpha_{\kappa} \Phi_{1}\left(l_{(\kappa)}\right)$ by $\tilde{\Phi}_{1}$ and $\sum_{\kappa=1}^{2^{n}} \alpha_{\kappa} \Phi_{2}\left(l_{(\kappa)}\right)$ by $\tilde{\Phi}_{2}$. Then, we conclude that $\tilde{\Phi}_{1}=$ $\operatorname{diag}\left\{\varepsilon_{1}, \ldots, \varepsilon_{n}\right\}$ and $\tilde{\Phi}_{2}=\operatorname{diag}\left\{1-\varepsilon_{1}, \ldots, 1-\varepsilon_{n}\right\}$, where $0 \leq \varepsilon_{1}, \ldots, \varepsilon_{n} \leq 1$. Thus, we have

$$
\tilde{\mathcal{A}}_{c}=\left(\begin{array}{cc}
\mathbf{0} & I_{n} \\
-\tilde{\Phi}_{1}\left(\mathcal{H}_{1}-k_{1} G\right)-k_{1} G & -\tilde{\Phi}_{1}\left(\mathcal{H}_{2}-k_{2} G\right)-k_{2} G
\end{array}\right)
$$

We can observe that $\tilde{\mathcal{A}}_{c}$ is a time varying matrix due to $\tilde{\Phi}_{i}$. If we denote $\Delta \mathcal{A}_{c}=\left(\begin{array}{cc}\mathbf{0} & \mathbf{0} \\ -\tilde{\Phi}_{1}\left(\mathcal{H}_{1}-k_{1} G\right) & -\tilde{\Phi}_{1}\left(\mathcal{H}_{2}-k_{2} G\right)\end{array}\right)$, then, $\tilde{\mathcal{A}}_{c}=\mathcal{A}_{c}+\Delta \mathcal{A}_{c}$. The system (10) is asymptotically stable if we have $\tilde{\mathcal{A}}_{c}^{T} \mathcal{P}+\mathcal{P} \tilde{\mathcal{A}}_{c} \prec 0$. Since we already know that the eigenvalues of matrix $\mathcal{A}_{c}^{T} \mathcal{P}+\mathcal{P} \mathcal{A}_{c} \prec 0$, we should verify that $\left\|\Delta \mathcal{A}_{c}^{T} \mathcal{P}+\mathcal{P} \Delta \mathcal{A}_{c}\right\|$ is sufficiently small to guaranty that the origin of $\dot{\mathrm{e}}=\tilde{\mathcal{A}}_{c} \mathrm{e}$ is asymptotically stable. In other words, the stability of (10) could not be global.

Lemma 1: [13] Let $A$ be Hermitian, $X \in \mathbb{C}^{n \times k}$ have full column rank, and $M \in \mathbb{C}^{k \times k}$ be Hermitian having eigenvalues $\mu_{1} \leq \mu_{2} \leq \cdots \leq \mu_{k}$. Set $R=A X-X M$. There exist $k$ eigenvalues $\lambda_{i_{1}} \leq \lambda_{i_{2}} \leq \cdots \leq \lambda_{i_{k}}$ of A such that the following inequalities hold. Note that the subset $\left\{\lambda_{i_{j}}\right\}_{j=1}^{k}$ may be different at different occurrences. Then,

$$
\max _{1 \leq j \leq k}\left|\mu_{j}-\lambda_{i_{j}}\right| \leq \frac{\|R\|_{2}}{\xi_{\min }(X)} .
$$

Note that we use $\xi_{\min }(\cdot)$ to represent the minimum singular value of matrix inside the parenthesis. The notation $\|\cdot\|_{2}$ represents the induced 2-norm, which is defined by $\|A\|_{2}=$ $\sqrt{\lambda_{\max }\left(A^{*} A\right)}$, where $A^{*}$ is the conjugate transpose of $A$.

A quadratic Lyapunov function $V=\mathrm{e}^{T} \mathcal{P} \mathrm{e}$ is selected to prove the stability of the overall system. Then, we can obtain

$$
\dot{V}=\dot{\mathrm{e}}^{T} \mathcal{P} \mathrm{e}+\mathrm{e}^{T} \mathcal{P} \dot{\mathrm{e}}=\mathrm{e}^{T}\left(\tilde{\mathcal{A}}_{c}^{T} \mathcal{P}+\mathcal{P} \tilde{\mathcal{A}}_{c}\right) \mathrm{e}
$$

To ensure that the system (10) is asymptotically stable, we have to make sure that $\tilde{\mathcal{A}}_{c}^{T} \mathcal{P}+\mathcal{P} \tilde{\mathcal{A}}_{c} \prec 0$, in other words, 
$\lambda_{\max }\left(\tilde{\mathcal{A}}_{c}^{T} \mathcal{P}+\mathcal{P} \tilde{\mathcal{A}}_{c}\right)<0$. Since $\Delta \mathcal{A}_{c}^{T} \mathcal{P}+\mathcal{P} \Delta \mathcal{A}_{c}=\left(\tilde{\mathcal{A}}_{c}^{T} \mathcal{P}+\right.$ $\left.\mathcal{P} \tilde{\mathcal{A}}_{c}\right)-\left(\mathcal{A}_{c}^{T} \mathcal{P}+\mathcal{P} \mathcal{A}_{c}\right)$ and according to lemma 1 , we have

$$
\left|\lambda_{\max }\left(\mathcal{A}_{c}^{T} \mathcal{P}+\mathcal{P} \mathcal{A}_{c}\right)-\lambda_{\max }\left(\tilde{\mathcal{A}}_{c}^{T} \mathcal{P}+\mathcal{P} \tilde{\mathcal{A}}_{c}\right)\right| \leq\left\|\Delta \mathcal{A}_{c}^{T} \mathcal{P}+\mathcal{P} \Delta \mathcal{A}_{c}\right\|_{2}
$$

Therefore, system (10) is asymptotically stable, if

$$
\left\|\Delta \mathcal{A}_{c}^{T} \mathcal{P}+\mathcal{P} \Delta \mathcal{A}_{c}\right\|_{2} \leq\left|\lambda_{\max }\left(\mathcal{A}_{c}^{T} \mathcal{P}+\mathcal{P} \mathcal{A}_{c}\right)\right|
$$

According to the property of 2-norm, we have $\| \Delta \mathcal{A}_{c}^{T} \mathcal{P}+$ $\mathcal{P} \Delta \mathcal{A}_{c}\left\|_{2} \leq\right\| \Delta \mathcal{A}_{c}^{T} \mathcal{P}\left\|_{2}+\right\| \mathcal{P} \Delta \mathcal{A}_{c}\left\|_{2}=2\right\| \mathcal{P} \Delta \mathcal{A}_{c} \|_{2}$. If we rewrite $\Delta \overline{\mathcal{A}}_{c}$ as follows

$$
\Delta \mathcal{A}_{c}=\left(\begin{array}{cc}
\mathbf{0} & \mathbf{0} \\
\mathbf{0} & \tilde{\Phi}_{1}
\end{array}\right) \cdot\left(\begin{array}{cc}
\mathbf{0} & \mathbf{0} \\
-\mathcal{H}_{1}+k_{1} G & -\mathcal{H}_{2}+k_{2} G
\end{array}\right)
$$

knowing that $\left\|\left(\begin{array}{cc}\mathbf{0} & \mathbf{0} \\ \mathbf{0} & \tilde{\Phi}_{1}\end{array}\right)\right\|_{2}=\left\|\tilde{\Phi}_{1}\right\|_{2}$ and the 2-norm is used, we obtain

$$
\left\|\Delta \mathcal{A}_{\mathcal{C}}\right\|_{2} \leq\left\|\tilde{\Phi}_{1}\right\|_{2} \cdot\left\|\left(\begin{array}{cc}
\mathbf{0} & \mathbf{0} \\
-\mathcal{H}_{1}+k_{1} G & -\mathcal{H}_{2}+k_{2} G
\end{array}\right)\right\|_{2}
$$

Therefore, if $\left\|\tilde{\Phi}_{1}\right\|_{2} \neq 0$, inequality (15) can be rewritten as follows

$$
\left\|\left(\begin{array}{cc}
\mathbf{0} & \mathbf{0} \\
-\mathcal{H}_{1}+k_{1} G & -\mathcal{H}_{2}+k_{2} G
\end{array}\right)\right\|_{2} \leq \frac{\left|\lambda_{\max }\left(\mathcal{A}_{c}^{T} \mathcal{P}+\mathcal{P} \mathcal{A}_{c}\right)\right|}{2 \lambda_{\max }(\mathcal{P})\left\|\tilde{\Phi}_{1}\right\|_{2}}
$$

Specifically, $\left\|\tilde{\Phi}_{1}\right\|_{2}=0$ means that the controller (12) is unsaturated for all $i \in \mathcal{V}$. Then, the stability is directly obtained if $G$ is positive definite.

Thus, the system (10) is asymptotically stable, if inequality (16) is satisfied.

Now we recall the procedure of the controller design as follows.

- Design the gain matrix $\left[\begin{array}{ll}k_{1} & k_{2}\end{array}\right]$ of the system without saturations. Once $k_{1}$ and $k_{2}$ are selected, the right-hand side of (16) is fixed.

- Select matrix $\mathcal{H}$ satisfying (16).

- Calculate the invariant set $\Omega(\mathcal{P}, \rho)$, which satisfies $\Omega(\mathcal{P}, \rho) \subseteq\left\{\mathrm{e} \in R^{2 n}:\left|H_{i} \mathrm{e}\right| \leq b, i \in \mathcal{V}\right\}$.

- Verify that the initial condition satisfies e $\left(t_{0}\right) \in \Omega(\mathcal{P}, \rho)$.

The region of attraction $\Omega(\mathcal{P}, \rho)$ of the origin of (14) is shown in Fig. 2.

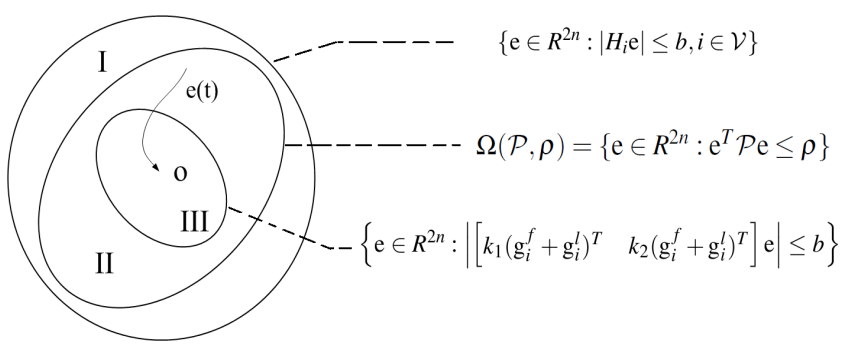

Fig. 2. The region of attraction $\Omega(\mathcal{P}, \rho)$ is shown in region II. Region I represents the region where inequality (16) is satisfied. Region III represents the region where the control inputs $u_{1}, \ldots, u_{2}$ are all unsaturated.

Thus, we obtain that when the initial condition satisfies $\mathrm{e}\left(t_{0}\right) \in \Omega(\mathcal{P}, \rho)$, the origin of (14) is asymptotically stable.

\section{B. Switching topology}

In this paper, we focus on the formation of the UAVs with limited sensing range. One UAV can sense the UAVs in its neighborhood. The switch occurs when there are UAVs that enter or escape the neighborhood of a UAV. Additionally, the change of leader will also cause the switch of topology. We treat this kind of switching as "arbitrary switching". The existence of a common Lyapunov function for all subsystems is a necessary and sufficient condition for a switching system to be asymptotically stable under arbitrary switching [14].

$$
\mathcal{A}_{c, \gamma}=\left(\begin{array}{cc}
\mathbf{0} & I_{n} \\
-k_{1} G_{\gamma} & -k_{2} G_{\gamma}
\end{array}\right)
$$

The objective is to find such a matrix $\mathcal{P}$, which satisfies

$$
\mathcal{A}_{c, \gamma}^{T} \mathcal{P}+\mathcal{P} \mathcal{A}_{c, \gamma} \prec 0, \gamma=0,1, \ldots
$$

We rewrite it as

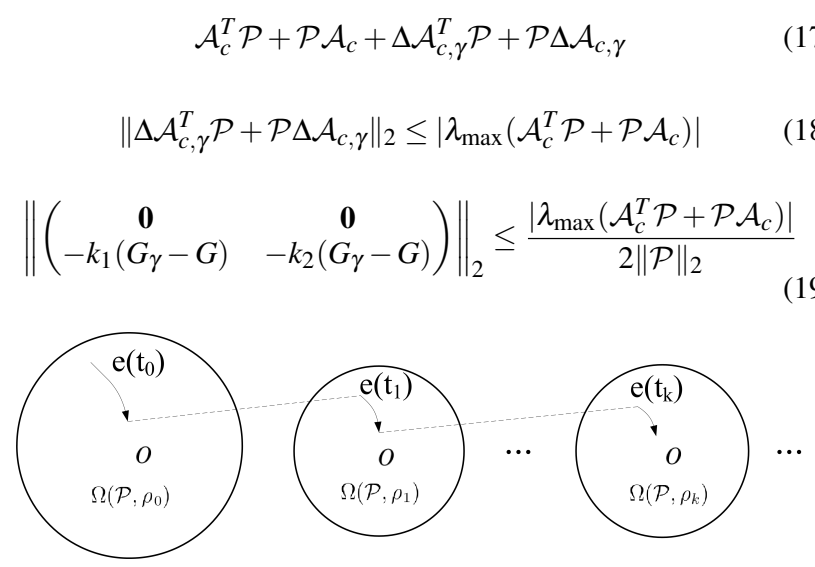

Fig. 3. The circles represent the estimated region of attraction for the time intervals in $\Gamma$. The letter " $o$ " represents the origin.

For a formation with switching topology, the interaction matrices are represented by $G_{\gamma} \in \Gamma$. Then, we observe that the closed-loop system (14) is a switching system. The common Lyapunov function is selected as $V=\mathrm{e}^{T} \mathcal{P}$. Then, the derivative of the common Lyapunov function $V$ is negative semi-definite, if the following condition is satisfied

$$
\mathrm{e}\left(t_{\gamma}\right) \in \Omega\left(\mathcal{P}, \rho_{\gamma}\right) \quad \gamma=0,1, \ldots
$$

where $t_{\gamma}$ represents the time instance of the $\gamma$-th switching (shown in Fig.1). Then, the L-F formation with switching topology is stable if (20) is satisfied.

\section{Simulation}

Heudiasyc laboratory has developed a PC-based simulatorexperiment framework for controlling a quadrotor and also a flock of quadrotors. The quadrotors used are the ones, manufactured by Parrot, as shown in Fig.4. The programs (written in $\mathrm{C}++$ ) running in the UAVs are the same, both in the simulator and in the embedded processors of real UAVs. When we realize the real-time experiment, the PC acts as a ground station, which is responsible for displaying and sending instructions such as taking off and landing. The UAVs are all autonomous. Once the formation is started, 
the program runs on-board each UAV. There does not exist a central controller that sends control signals to the UAVs. Therefore, the formation program is launched in a distributed way. This framework permits the simulation to reflect better the real-time experiment, where the ArDrone2 quadrotors manufactured by Parrot are used. The motion capture system Optitrack is used to localize the UAVs in the formation.

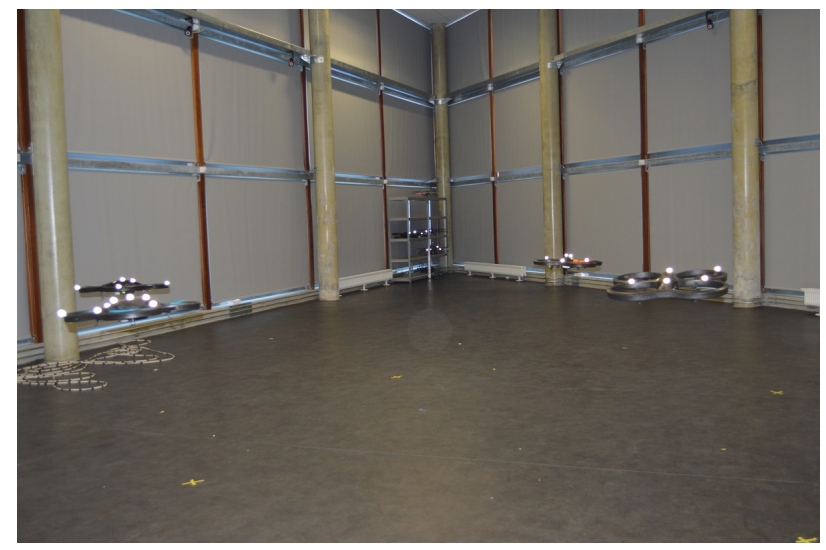

Fig. 4. The formation of quadrotors

A formation of four UAVs is shown in Fig.5. The initial coordinates of the UAVs are given as follows: UAV 1 $(-1.3,1.1,0)$, UAV $2(-1,-1,0)$, UAV $3(1,-1,0)$ and UAV $4(1.2,0.8,0)$. The formation task of the UAVs is to move towards a destination point while maintaining desired inter-distances.

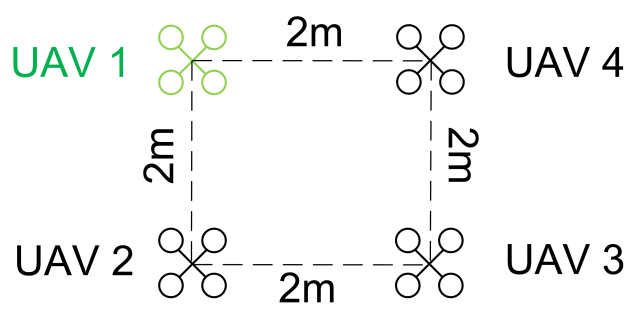

Fig. 5. The desired formation pattern of four quadrotors

We suppose that at time $t_{1}$, the edges $(2,3)$ and $(3,2)$ are broken. The interaction matrix becomes singular (see $G_{1}$ in (21)). We assign UAV 3 as a new leader in the formation. Then, there are two leaders in the formation such that the interaction matrix is invertible (see $G_{2}$ in (21)).

$$
G_{1}=\left[\begin{array}{cccc}
2 & -1 & 0 & 0 \\
-1 & 1 & 0 & 0 \\
0 & 0 & 1 & -1 \\
0 & 0 & -1 & 1
\end{array}\right] \quad G_{2}=\left[\begin{array}{cccc}
2 & -1 & 0 & 0 \\
-1 & 1 & 0 & 0 \\
0 & 0 & 2 & -1 \\
0 & 0 & -1 & 1
\end{array}\right]
$$

We give two simulation results using MATLAB as follows. In Fig. 6, the leader is always the UAV 1, while in Fig.7, UAV 3 is assigned as a new leader as soon as the edges $(2,3)$ and $(3,2)$ are broken.
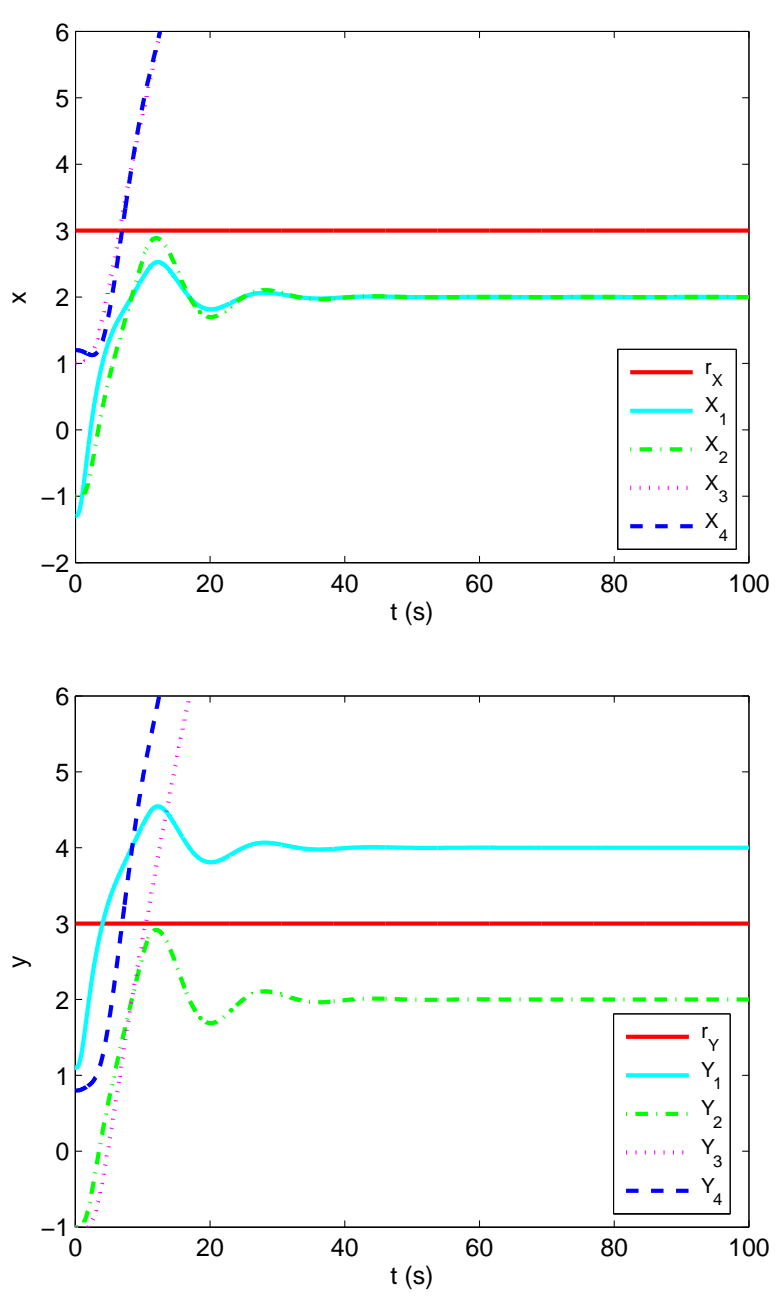

Fig. 6. Formation of UAVs with edges $(2,3)$ and $(3,2)$ broken at $t_{1}=10 \mathrm{~s}$.

We observe that in Fig.6, the UAVs 3 and 4 can not aggregate around the desired point, since the interaction matrix has been singular after $t_{1}$. However, in Fig.7, a new assigned leader makes the interaction matrix invertible after $t_{1}$, therefore, the formation task is accomplished.

The foregoing simulation results are illustrated using our simulator-experiment framework. In the real-time experiment, a formation of four quadrotors is shown. The objective is to track a rectangular trajectory. The video of the simulator animation and the real-time experiment is given on the site https://youtu.be/Pd_kZKqo66I.

\section{CONCLUSION}

In this paper, a distributed neighbor-based formation control is proposed for the multi-UAV systems with L-F structure. The approximately decoupled translational dynamics are obtained, owing to the employment of saturation function. The consensus conditions of the multi-UAV system for both fixed and switching topology are detailed. Simulations 

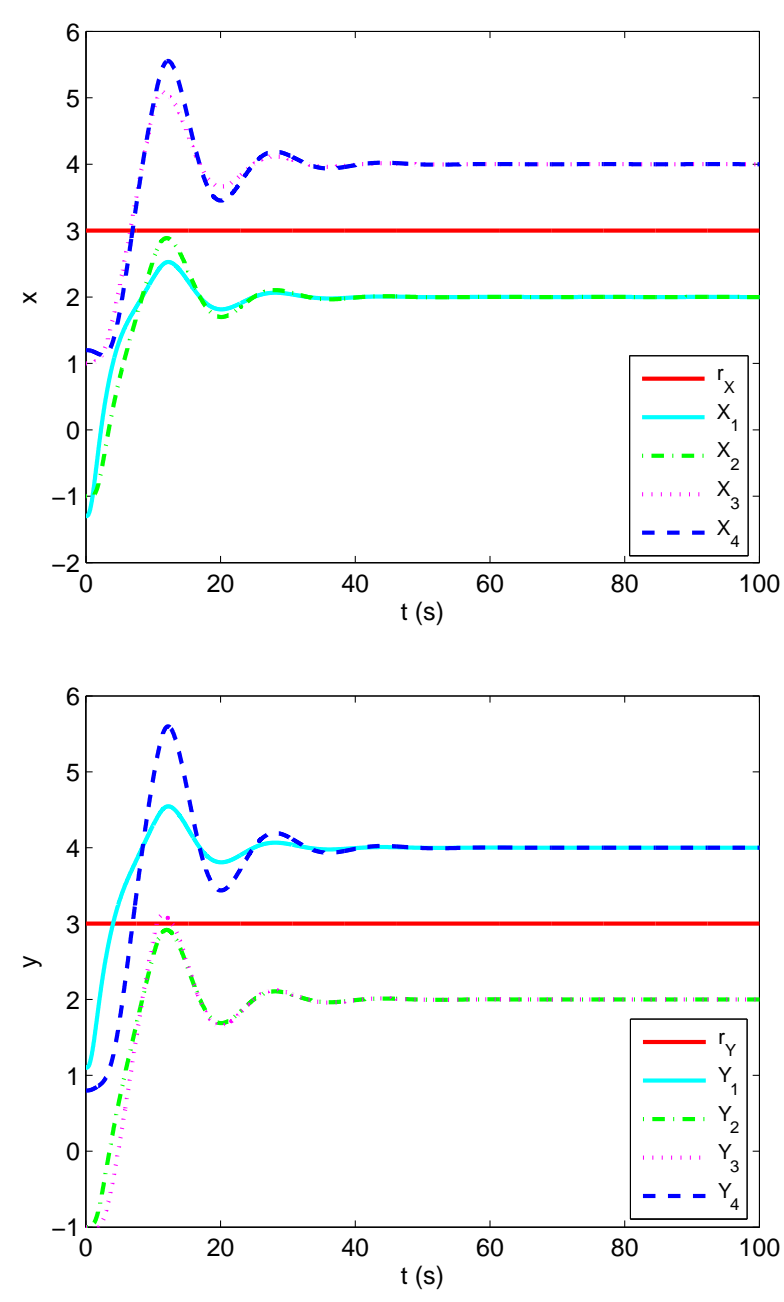

Fig. 7. Formation of UAVs with edges $(2,3)$ and $(3,2)$ broken at $t_{1}=10$ s. UAV 3 is assigned as a new leader after $t_{1}$.

and real-time experiments show the satisfactory performance of such a strategy.

\section{ACKNOWLEDGMENT}

We would like to extend my gratitude to my colleague Guillaume Sanahuja who is engineer in Heudiasyc laboratory. He has developed such a respected work on the simulator and real-time experiment platform, which permits us to implement our algorithm on it.

\section{REFERENCES}

[1] Panagou, D., Kumar, V., "Maintaining visibility for leader-follower formations in obstacle environments," 2012 IEEE International Conference on Robotics and Automation (ICRA), pp.1811-1816, 2012.

[2] V. Roldao, R. Cunha, D. Cabecinhas, C. Silvestre, P. Oliveira, "A leader-following trajectory generator with application to quadrotor formation flight," Robotics and Autonomous Systems, vol.62, iss.10, 2014.

[3] A. Kushleyev, D. Mellinger, V. Kumar, "Towards A Swarm of Agile Micro Quadrotors," Autonomous Robots, 35(4):287-300, 2013.
[4] Ni, W., Cheng D., "Leader-following consensus of multi-agent systems under fixed and switching topologies," Systems \& Control Letters, vol.59, iss.3-4, pp.209-217, 2010.

[5] Hong, Y., Hu, J., Gao, L., "Tracking control for multi-agent consensus with an active leader and variable topology," Automatica, vol.42, iss.7, pp.1177-1182, 2006.

[6] Olfati-Saber, R., Murray, R.M., "Consensus problems in networks of agents with switching topology and time-delays," IEEE Transactions on Automatic Control, vol.49, no.9, pp.1520-1533, 2004.

[7] Ren W., Beard, R.W., Atkins, E.M., "Information consensus in multivehicle cooperative control," IEEE Control Systems Magazine, vol.27, no.2, pp.71-82, 2007.

[8] Hong, Y., Gao, L., Cheng, D., Hu, J., "Lyapunov-Based Approach to Multiagent Systems With Switching Jointly Connected Interconnection," IEEE Transactions on Automatic Control, vol.52, no.5, pp.943948, May 2007.

[9] Fax, J.A, Murray, R.M., "Information flow and cooperative control of vehicle formations," IEEE Transactions on Automatic Control, vol.49, no.9, pp.1465-1476, 2004.

[10] Gonzalez-Vazquez, S., Moreno-Valenzuela, J.: 'Motion Control of a Quadrotor Aircraft via Singular Perturbations', International Journal of Advanced Robotic Systems, 10(368):1-16,2013.

[11] Bresciani, T., Modelling, "Identification and Control of a Quadrotor Helicopter, Master thesis, Lund University, 2008.

[12] Bateman, A., Lin Z., "An analysis and design method for discretetime linear systems under nested saturation," IEEE Transactions on Automatic Control, vol.47, no.8, pp.1305-1310, 2002.

[13] Leslie Hogben, "Handbook of Linear Algebra," CRC Press, 2007.

[14] Jun Zhao, David J. Hill, "On stability, $L_{2}$-gain and $H_{\infty}$ control for switched systems," Automatica, vol.44, iss.5, pp.1220-1232, 2008.

[15] Hou, Z., Fantoni, I., and Zavala-Rio, A., "Modeling and Decentralized Control for the Multiple UAVs Formation based on Lyapunov design and redesign," IFAC Workshop RED-UAS, Compiègne, France, 2013.

[16] Hou, Z., Fantoni, I.,"Distributed Leader-Follower Formation Control for Multiple Quadrotors with Weighted Topology," 10th International Conference on System of Systems Engineering (SoSE), San Antonio, 2015. 